\section{Beta-3-Agonist bewährt sich bei überaktiver Blase}

\author{
Der Beta-3-Rezeptor-Agonist Mirabegron ist eine neue Substanz zur \\ Behandlung der überaktiven Blase. Jetzt wurden zeitgleich zwei \\ Phase-III-Studien veröffentlicht, in denen die Wirksamkeit und \\ Verträglichkeit des Wirkstoffs untersucht wurden.
}

$\mathrm{V}$ on einer Therapie mit antimuskarinergen Medikamenten profitieren viele Patienten mit überaktiver Blase nicht ausreichend, auch können für manche der Betroffenen unerwünschte Wirkungen solcher Medikamente wie trockener Mund sehr belastend sein. Eine neue Option zur symptomatischen Behandlung könnte Mirabegron sein. Das Medikament wirkt agonistisch am Beta-3-adrenergen Rezeptor und entspannt dadurch die Blasenmuskulatur.

In der Phase-III-Studie von Khullar et al. wurde der Mirabegron gegen Placebo und gegen eine Standardtherapie mit antimuskarinerg wirkendem Tolterodin geprüft. An dieser Studie hatten knapp 2.000 Männer und Frauen mit überaktiver Blase teilgenommen. Jeweils ein Viertel erhielt zwölf Wochen lang entweder Placebo, Mirabegron $50 \mathrm{mg} / \mathrm{d}$, Mirabegron $100 \mathrm{mg} / \mathrm{d}$ oder retardiertes Tolterodin ( $4 \mathrm{mg} / \mathrm{d})$. Alle Teilnehmer wurden vor Studienbeginn zwei Wochen lang auf Placebo eingestellt. Etwas mehr als die Hälfte der Patienten berichtete über Probleme mit der Kontinenz. Patienten mit Stressinkontinenz waren allerdings ausgeschlossen worden. Die Hälfte der Patienten war zuvor schon medikamentös gegen Miktionsprobleme behandelt worden, zwei Drittel hatten die vorhergehende Therapie wegen mangelnder Wirksamkeit abgebrochen, $27 \%$ wegen unerwünschter Wirkungen.

Mit Beginn der Studienmedikation hatten die Patienten im Schnitt 11,6 Miktionen pro Tag in einem Tagebuch notiert. Mit Placebo war diese Zahl nach zwölf Wochen im Schnitt um 1,3 Miktionen gesunken, mit Mirabegron $50 \mathrm{mg} / \mathrm{d}$ um 1,9, mit $100 \mathrm{mg} / \mathrm{d}$ um 1,8 Miktionen und mit Tolterodin um 1,6 Miktionen. Mit Mirabegron waren die Unterschiede im Vergleich zu Placebo signifikant, nicht so mit Tolterodin. Ein ähnliches Bild ergab sich bei der Zahl der Inkontinenzepisoden. $\mathrm{Zu}$ Therapiebeginn hat- ten Patienten mit Inkontinenz im Schnitt etwa 2,7 solcher Episoden pro Tag erwähnt, mit Placebo sank der Wert im Schnitt um 1,2 Episoden, mit 50 mg Mirabegron um 1,6 und mit $100 \mathrm{mg}$ um 1,5 Episoden. Kaum Änderungen gab es bei einer Therapie mit Tolterodin (minus 1,3 Episoden pro Tag). Die Rate der unerwünschten Wirkungen mit der neuen Arznei lag auf Placeboniveau, vor allem kam es im Gegensatz zur Tolterodintherapie nicht häufiger als mit Placebo zu Mundtrockenheit. Auffällig war eine leichte, klinisch aber unbedeutende Erhöhung der Pulsrate.

Eine vergleichsweise gute Wirksamkeit wurde auch in der Studie von Chapple et al. festgestellt. Die Forscher hatten über ein ganzes Jahr Mirabegron in der 50und 100-mg-Dosierung gegen Tolterodin (4 mg/d) geprüft. Die knapp 2.450 Patienten hatten im Schnitt elf Miktionen pro Tag. Mit Mirabegron $50 \mathrm{mg}$ war die Zahl nach zwölf Monaten um 1,3 Episoden und mit der 100-mg-Dosierung um 1,4 Episoden gesunken. Die Therapie war in dieser Studie damit ähnlich gut wirksam wie mit Tolterodin (ebenfalls minus 1,4 Episoden). Dies zeigte sich auch bei der Zahl der Inkontinenzepisoden: Sie ging mit der 50-mg-Dosierung im Schnitt um 1,0 und mit $100 \mathrm{mg} / \mathrm{d}$ um 1,2 Episoden zurück; mit Tolterodin gelang ebenfalls eine Reduktion um 1,2 Episoden. Auch der Anteil der Patienten mit einem Rückgang der Inkontinenzepisoden um mindestens die Hälfte war mit der 100-mgDosierung ähnlich hoch wie mit Toltero$\operatorname{din}(66,3 \%$ vs. $66,8 \%)$ und etwas niedriger mit $50 \mathrm{mg}$ Mirabegron (63,7\%). Komplett kontinent wurden $43,4 \%$ mit $50 \mathrm{mg} / \mathrm{d}$ des Beta-3-Agonisten und 45,8\% mit $100 \mathrm{mg} / \mathrm{d}$ sowie $45,1 \%$ mit Tolterodin. Bei der Wirksamkeit lag also zumindest die 100-mg-Dosierung der neuen Arznei auf einem vergleichbaren Niveau wie die antimuskarinerge Standardtherapie, es brachen etwas weniger Patienten die Therapie wegen mangelnder Wirksamkeit ab als mit Tolterodin $(3,6 \%$ vs. $5,5 \%)$.

Bei den Nebenwirkungen war die Rate von Patienten, die über Mundtrockenheit klagten, mit Mirabegron erwartungsgemäß deutlich geringer als mit der Standardtherapie ( $2,3 \%$ vs. $8,6 \%)$. Insgesamt waren die Anteile von Patienten mit moderaten und schweren unerwünschten Wirkungen in den drei Gruppen ähnlich hoch, auch die Rate der Therapieabbrüche war mit etwa $6 \%$ in allen drei Gruppen vergleichbar. Mit Mirabegron $100 \mathrm{mg}$ stellten die Studienärzte eine leicht erhöhte Pulsrate fest (plus 1,3 bis 1,6 Schläge pro Minute), dies war allerdings auch in der Gruppe mit Tolterodin der Fall (plus 1,5 bis 1,9 Schläge pro Minute).

Fazit: Mirabegron lindert nach diesen Studiendaten in einer Dosis von 100 $\mathrm{mg} / \mathrm{d}$ die Symptome bei überaktiver Blase ähnlich gut wie eine antimuskarinerge Standardtherapie, vermeidet aber eine für die Patienten oft lästige Mundtrockenheit. In den USA ist die Arznei bereits seit Juli 2012 auf dem Markt. Von der europäischen Arzneimittelagentur EMA gibt esbereits ein positives Votum, die Zulassung wird noch in diesem Jahr erwartet.

Thomas Müller

Khullar $\mathrm{V}$ et al. Efficacy and Tolerability of Mirabegron, a $\beta_{3}$-Adrenoceptor Agonist, in $\mathrm{Pa}$ tients with Overactive Bladder: Results from a Randomised European-Australian Phase 3 Trial. Eur Urol 2013, 63: 283-95

Chapple CR et al. Randomized Double-blind, Active-controlled Phase 3 Study to Assess 12-Month Safety and Efficacy of Mirabegron, a $\beta_{3}$-Adrenoceptor Agonist, in Overactive Bladder. Eur Urol 2013, 63: 296-305 